\title{
Análisis psicométrico del Cuestionario de Autorregulación del Aprendizaje en estudiantes de Psicología de una universidad privada de Arequipa
}

\author{
Psychometric analysis of Learning Self-regulation Questionnaire in \\ Psychology students from a private university at Arequipa \\ Walter L. Arias Gallegos ${ }^{1}$, Renzo Rivera ${ }^{2}$, Karla D. Ceballos Canaza ${ }^{3}$ \\ Universidad Católica San Pablo, Arequipa, Perú
}

Recibido: $07-05-20$

Aceptado: $12-06-20$

\section{Resumen}

En el presente estudio de corte psicométrico, se analiza la validez y confiabilidad del Cuestionario de Autorregulación del Aprendizaje en una muestra de 151 estudiantes universitarios de la carrera de Psicología de una universidad privada de la ciudad de Arequipa. Se realizaron los cálculos con el análisis factorial exploratorio y el método de consistencia interna, para la validez y la confiabilidad, respectivamente. Los resultados indican que la prueba tiene una estructura de dos factores, similar a la reportada en estudios previos que han confirmado la estructura bifactorial de la versión original.

Palabras clave: Autorregulación del aprendizaje; psicometría; análisis factorial exploratorio; confiabilidad.

\begin{abstract}
In the present study, psychometrical sort of, we analyze the validity and reliability of the Learning Self-Regulated Questionnaire in a sample of 151 university students of the professional career of Psychology from a private university at Arequipa City. We calculate validity and reliability through exploratory factor analysis and internal consistency method, respectively. The results indicate that the instrument has a two factors structure, similar to previous studies that had confirmed the bifactorial structure of the original version.
\end{abstract}

Keywords: Self-regulated learning; psychometrics; exploratory factorial analysis; reliability.

\footnotetext{
${ }^{1}$ Docente Universidad Católica San Pablo. Autor para correspondencia. E-mail: warias@ucsp.edu.pe ORCID: https://orcid.org/0000-0002-4183-5093

${ }^{2}$ Docente Universidad Católica San Pablo, Arequipa, Perú. E-mail: crrivera@ucsp.edu.pe ORCID: https://orcid.org/0000-0002-5897-9931

${ }^{3}$ Estudiante Universidad Católica San Pablo, Arequipa, Perú. E-mail: Karla.ceballos@ucsp.edu.pe ORCID: https://orcid.org/0000-0001-8006-3738
}

\footnotetext{
(C) Los autores. Este artículo es publicado por la Revista de Investigación en Psicología de la Facultad de Psicología, Universidad Nacional Mayor de San Marcos. Este es un artículo de acceso abierto, distribuido bajo los términos de la licencia Creative Commons Atribucion - No Comercia_Compartir Igual 4.0 Internacional. (http://creativecommons.org/licenses/by-nc-sa/4.0/) que permite el uso no comercial, distribución y reproducción en cualquier medio, siempre que la obra original sea debidamente citada.
} 
La autorregulación del aprendizaje es una variable esencial para la cognición porque determina formas más profundas y reflexivas de aprendizaje (Escurra, 2002), además de encontrarse motivadas por factores intrínsecos y orientarse según los propios intereses, conocimientos y metas académicas del estudiante (Valle, Cabanach, Rodríguez, Núñez y González-Pineda, 2006). En ese sentido, uno de los retos más importantes de la educación debe ser la promoción del autoaprendizaje que está mediado por las habilidades metacognitivas básicas para aprender a aprender (Rodríguez y Vázquez, 2013).

La autorregulación del aprendizaje empero, se va formando desde el nacimiento, siendo un hito fundamental, la interiorización del lenguaje que favorece el desarrollo de las habilidades cognitivas y la autorregulación de la conducta (Vigotsky, 1979). Este proceso tiene su correlato fisiológico en la maduración de las zonas frontales del cerebro, particularmente de la porción dorsolateral de la corteza prefrontal (Arias y Vilca, 2007), y se fortalece con la estimulación sociocultural que recibe el niño de los adultos que le rodean, que constituye lo que Vigotsky llamó zona de desarrollo próximo (Vigotsky, 1995). De este modo, el acompañamiento de los adultos o de personas más adelantas (como sus compañeros de clase), conforma un andamiaje que, al ser retirado paulatina y sistemáticamente, tienen por resultado la capacidad de autorregularse.

Estudios experimentales en niños, han determinado que la capacidad de autorregular la conducta tiene efectos positivos durante la adolescencia y la edad adulta, favoreciendo el control inhibitorio y la planificación de las acciones (Gómez y Luciano, 1991). Asimismo, se ha visto que el género es un factor interviniente en el desarrollo de la autorregulación conductual, pues dado que el cerebro de las niñas se lateraliza antes de la pubertad, las mujeres suelen tener una mejor capacidad autorregulatoria más tempranamente que los varones (Parra, Cerda, López-Vargas y Saiz, 2014). La socialización es otro factor importante, pues el trabajo en grupo o simplemente, las relaciones con otras personas, promueven habilidades de interacción social que ponen en juego capacidades cognitivas y metacongitivas (Wertsch, 1988).

De hecho, algunos estudios muestran que la interacción con los padres tiene efectos en la autorregulación emocional de los niños, siendo fundamentales los etilos de crianza (Covarrubias, Gómez y Estrevel, 2005). En ese sentido, la autorregulación del aprendizaje tiene componentes cognitivos y motivacionales que implican a la emoción (Valle, González, Barca y Núñez, 2000), de modo que, en buena cuenta, la autorregulación del aprendizaje es una autorregulación de la emoción, ya que son los afectos los que actúan como motivadores de la conducta (Gargurevich, 2008). Pero, tanto la afectividad como la cognición son estructurados históricamente en un contexto sociocultural determinado (Rodríguez, 2013). De este modo, la historia personal del individuo aporta a la autorregulación del aprendizaje a través del autocontrol y el autoconcepto (Fernandes, Marín y Urquijo, 2010). 
En el campo educativo, la autorregulación del aprendizaje se relaciona con la motivación (Lamas, 2008), la autoeficacia académica (Alegre, 2014), con los estilos de aprendizaje (Contreras y Lozano, 2012), la metacognición (Vallejos, Jaimes, Aguilar y Merino, 2012) y el rendimiento académico (Fuente, Pichardo, Justicia y Berbén, 2008). Sin embargo, las relaciones entre estos constructos son complejas. En primer lugar, es necesario que el aprendizaje se vincule con emociones positivas a través de actividades didácticamente planificadas, pero no coercitivas (Merchán, Bermejo, \& González, 2014). Ello despertará la motivación de logro académico en los estudiantes, que es un potente motivador del aprendizaje (Thornberry, 2003). Pero se debe tener cuidado de que el estudiante esté verdaderamente motivado por aprender, pues la motivación de logro puede vincularse con otros aspectos como el prestigio académico, y otros reforzadores externos como las notas y los elogios (Valle, Cabanach, Rodríguez, Núñez y González-Pineda, 2006).

Por ello, es importante tener en cuenta los enfoques de aprendizaje que tienen los estudiantes (Valle, González, Núñez, Suárez, Piñeiro y Rodríguez, 2000), ya que, si presentan un enfoque superficial, no se esforzarán por aprender sino por aprobar, y requerirán de mayores reforzadores externos de parte del profesor; en cambio, los estudiantes con un enfoque profundo están motivados intrínsecamente por comprender y dominar los temas que estudian, y el profesor es solamente un facilitador (Meza, 2013). Por esta razón, es importante promover estrategias adecuadas de aprendizaje que promuevan la autorregulación del aprendizaje (Revel y González, 2007).

Aunque existen múltiples estrategias de aprendizaje, y cada cual hace un aporte sustancial al aprendizaje, las estrategias metacognitivas son las que desarrollan el aprendizaje autoconsciente y autorregulado (Valle, Barca, González y Núñez, 1999). Los estilos de aprendizaje también contribuyen a la autorregulación del aprendizaje, sobre todo los de tipo reflexivo o asimilador (Arias, 2014). Lamentablemente, los estudios realizados en Arequipa, han demostrado que en los estudiantes universitarios predominan los estilos de aprendizaje divergente y acomodadores, que ponen énfasis en los aspectos prácticos del aprendizaje en lugar de los contenidos teóricos, que son fundamentales para la comprensión y dominio del tema (Arias, 2011). Más aún, en un estudio previo, se pudo constatar que los estudiantes de la carrera de Psicología de tres universidades de Arequipa, presentan niveles bajos de metacognición y que, ésta no tiene efecto en los estilos de aprendizaje que emplean (Arias, Zegarra y Justo, 2014).

En ese sentido, las metas académicas que se plantean los estudiantes son determinantes de la autorregulación del aprendizaje y sus enfoques de aprendizaje (Castillo, Balaguer y Duda, 2001). En el estudio de González (2013) por ejemplo, se encontró que, en 289 estudiantes de psicología de Lima, las concepciones de aprendizaje que manifestaron de manera predominante eran de tipo directo, lo que quiere decir que relacionan el estudio con los logros académicos, 
pero no se sienten responsables de sus propios aprendizajes, o, dicho de otro modo, no movilizan sus recursos internos que intervienen en el aprendizaje.

En el estudio de Martínez-Fernández (2007) en el que se evaluó a 276 estudiantes de Psicología en España, se reportó que los estudiantes de semestres iniciales tenían concepciones directas del aprendizaje, mientras que los de los semestres finales tenían concepciones constructivas. Esto supone que los estudiantes al inicio de la carrera carecen de estrategias metacognitivas y que conforme avanzan, las van desarrollando. En un estudio sobre los estilos de aprendizaje con universitarios de cuatro carreras profesionales de Arequipa, encontramos que el estilo divergente era más predominante en los primeros semestres, pero en los últimos semestres, los estudiantes con estilos asimilador habían aumentado, aunque no llegaron a ser la mayoría (Arias, 2011). En ese sentido, es a través de la metacognición que los estilos de aprendizaje y las estrategias de aprendizaje se conectan (García, Sánchez, Jiménez y Gutiérrez, 2012), de ahí que la autorregulación forme parte de la metacognición (Vallejos, Jaimes, Aguilar y Merino, 2012). Por ello, es importante evaluar la metacognición de los estudiantes, sobre todo en el nivel universitario que es cuando deberían autorregular mejor su aprendizaje (Arias, 2008).

Ahora bien, con la finalidad de valorar todas estas variables que están implicadas en el aprendizaje autorregulado, se han diseñado y/o validado una amplia variedad de pruebas psicológicas en muestras de estudiantes universitarios peruanos, pero básicamente limeños, como la Prueba de Motivación de Logro Académico (Thornberry, 2003), el Inventario de Estrategias de Aprendizaje (Escurra, 2006), el Cuestionario de Clima de Aprendizaje (Matos, 2011), el Inventario de Estrategias Metacognitivas (Vallejos, Jaimes, Aguilar y Merino, 2012), el Cuestionario de Honey y Alonso de estilos de Aprendizaje (Escurra, 2011), etc.

En cuanto a la autorregulación del aprendizaje una prueba relacionada es el Cuestionario de Autorregulación Emocional de Gross y Thompson, que fue adaptado para el Perú por Gargurevich y Matos (2010). Ambos autores tradujeron este instrumento y lo validaron en 320 estudiantes universitarios de Lima, reportando una estructura bifactorial mediante el análisis factorial confirmatorio con buenos indicadores de ajuste y una confiabilidad superior a .7 para las dos dimensiones resultantes: reevaluación cognitiva y supresión emocional. Otro instrumento que evalúa la autorregulación conductual, particularmente del aprendizaje es el Cuestionario de Autorregulación del Aprendizaje Académico en la Universidad, que fue elaborado por Torre a partir del modelo teórico de Zimmerman y validado por Alegre (2014) en 284 estudiantes universitarios de Lima. En este estudio, se encontró que el cuestionario de 20 ítems presenta correlaciones ítem-test con valores entre .37 y .67 con una estructura unidimensional y un índice de confiabilidad de .862 calculado con la prueba Alfa de Cronbach. También se reportó que dicho instrumento presenta 
validez convergente con el Cuestionario de Autoeficacia Académica de Torre que se basa en los supuestos teóricos de Bandura.

Por último, el Cuestionario de Autorregulación de Aprendizaje de Williams y Deci (1996) ha sido validado por Matos (2009) en 369 estudiantes universitarios de Lima, reportándose una estructura de dos factores, Autonomía y Control (mediante el modelamiento de ecuaciones estructurales), tal y como sugieren los autores de la prueba, pero con índices de bondad de ajuste poco robustos (CFI=0.90). La autora también calculó la validez convergente, divergente y predictiva del cuestionario, así como su confiabilidad con valores de 0.79 y 0.78 , para cada una de sus dimensiones. Poco tiempo después, Chávez y Merino (2015) aplicaron este instrumento a 237 estudiantes de los primeros ciclos de estudios de una universidad privada de Lima, y confirmaron su estructura bifactorial con índices de bondad ajuste adecuados y una confiabilidad de 0.702 para la dimensión de Autonomía y de 0.744 para la dimensión de Control.

Cabe señalar que el Cuestionario de Autorregulación del Aprendizaje se basa en la teoría de la autodeterminación de Williams y Deci (1996) que plantea que las personas orientan su conducta en base a la autonomía que conducirá el compromiso por aprender. Sin embargo, los reactivos de la prueba no precisan diversos factores propios de la motivación académica y las estrategias metacognitivas (Thornberry, 2008), favoreciendo su superposición y limitando la discriminación de estos aspectos, que son esenciales para la valoración de las concepciones del aprendizaje de los estudiantes (Martínez-Fernández, 2007).

En tal sentido, el objetivo del presente estudio es analizar las propiedades psicométricas de la versión validada para Perú (Matos, 2009) del Cuestionario de Autorregulación del Aprendizaje, en una muestra de estudiantes de psicología de la ciudad de Arequipa. En consecuencia, el presente estudio es de tipo instrumental (Montero y León, 2002), y se siguieron los lineamientos de la teoría clásica de los test (Magnusson, 1969).

\section{MÉTODO}

\section{Muestra}

La muestra estuvo conformada por 155 estudiantes de la carrera de Psicología de una universidad privada de Arequipa. Debido a que cuatro personas no llenaron debidamente los instrumentos, la muestra se redujo a 151 estudiantes $(13.8 \%$ varones y $86.2 \%$ mujeres) con una edad media de 19.04 años y una desviación estándar de \pm 2.63 dentro de un rango de 16 a 34 años de edad. El $30.3 \%$ cursa estudios en el primer semestre, el 13.25 en segundo semestre, el $23.7 \%$ en tercer semestre, el $9.9 \%$ en cuarto semestre, el $12.5 \%$ en quinto semestre, el $2 \%$ en sexto semestre, el $3.9 \%$ en octavo semestre y el 3.9\% restante en décimo semestre. 
Los estudiantes fueron seleccionados de manera no probabilística mediante la técnica de grupos intactos.

\section{Instrumento}

El Cuestionario de Autorregulación del Aprendizaje (o Learning Self-Regulation Questionnaire) fue creado por Williams y Deci (1996) y consta de 14 ítems con alternativas de respuesta en una escala de Likert que va de 1 a 7, desde Nada verdadero para mí hasta Totalmente verdadero para mí. La versión original cuenta con dos dimensiones: la dimensión de Autonomía comprende los ítems 1, 3, 6, 9, 11 y 12; y la dimensión de Control comprende los ítems 2, 4, 5, 7, 8, 10, 13 y 14. Posee además validez de constructo, validez convergente y confiabilidad para cada una de sus dimensiones, con puntajes alfa de Cronbach de .78 para Autonomía y 0.70 para Control. Matos (2009) ha reportado validez de constructo obtenida mediante análisis factorial confirmatorio y niveles de confiabilidad adecuados para las dimensiones de Autonomía $(\alpha=.78)$ y Control $(\alpha=.79)$ en una muestra de 369 estudiantes universitarios de Lima, que cursaban estudios de Psicología, Ingeniería y Administración.

\section{Procedimiento}

Los datos fueron recogidos en las clases de la universidad en la que los estudiantes están matriculados en la carrera de Psicología. Los participantes fueron informados de los fines del estudio y se garantizó la confidencialidad de sus datos. Asimismo, se les reiteró la importancia de responder con sinceridad al instrumento y se les ofreció la oportunidad de conocer los resultados de manera individual si lo requerían.

\section{Análisis de datos}

Una vez recogida la información, se procedió al procesamiento estadístico mediante la teoría clásica de los tests (Livia y Ortiz, 2014), de modo que se obtuvieron los estadísticos descriptivos como media, desviación estándar, asimetría curtosis, el puntaje máximo y mínimo. También se realizaron correlaciones ítem-test para valorar la homogeneidad de la prueba y la confiabilidad se calculó mediante la prueba alfa de Cronbach (Kaplan, \& Saccuzzo, 2006), para lo cual se trabajó con el programa SPSS versión 20 (IBM, 2011). Para valorar la estructura interna de la prueba se aplicó el análisis factorial exploratorio (AFE) con el programa Factor versión 10.5.03 (Lorenzo-Seva, \& Ferranzo, 2017). Se utilizó el procedimiento de óptima implementación de análisis paralelo (Timmerman, \& Lorenzo-Seva, 2011) para la determinación del número de factores de la prueba, y teniendo en cuenta la cantidad de la muestra se utilizó el sesgo corregido y acelerado como técnica de análisis robusto (Lambert, Wildt \& Durand, 1991). Mientras que para la extracción de los factores se aplicó el método de mínimos cuadrados no ponderados robustos (RULS). 


\section{RESULTADOS}

En la Tabla 1 se tienen los estadísticos descriptivos donde se puede apreciar que los promedios de respuesta por ítem van de 1.821 para el ítem 2 ("Yo participo activamente en todas las clases porque otros pensarían mal de mí si no lo hiciera") hasta 5.503 para el ítem 11 (“La razón por la que continuaré ampliando mis conocimientos es porque es interesante aprender más"). Asimismo, las medidas de asimetría y curtosis son bajas pues se ubican dentro del rango $-1.5 /+1.5$, lo que sugiere que los ítems del Cuestionario de Autorregulación del Aprendizaje tienden hacia una distribución normal. También se pueden apreciar las correlaciones ítem test que tienen puntuaciones superiores a 0.4, siendo la más baja la del ítem $10(\mathrm{r}=0.408)$ y la más alta la del ítem $4(\mathrm{r}=0.628)$. los coeficientes de correlación sugieren que la prueba es homogénea porque todos los ítems miden el mismo constructo teórico.

\section{Tabla 1}

Estadisticos descriptivos de los items

\begin{tabular}{cccccccc}
\hline $\mathbf{N}^{\circ}$ Ítem & Mín. & Máx. & Media & D.E. & Asimetría & Curtosis & citc \\
\hline Ítem 1 & 1 & 7 & 3.854 & 1.674 & 0.251 & -0.613 & 0.433 \\
Ítem 2 & 1 & 7 & 1.821 & 1.249 & 1.966 & 4.045 & 0.533 \\
Ítem 3 & 1 & 7 & 4.218 & 1.491 & -0.064 & -0.796 & 0.597 \\
Ítem 4 & 1 & 7 & 2.933 & 1.668 & 0.534 & -0.770 & 0.628 \\
Ítem 5 & 1 & 7 & 4.615 & 1.552 & -0.376 & -0.450 & 0.625 \\
Ítem 6 & 1 & 7 & 5.079 & 1.363 & -0.545 & -0.297 & 0.612 \\
Ítem 7 & 1 & 7 & 2.397 & 1.527 & 1.055 & 0.322 & 0.561 \\
Ítem 8 & 1 & 7 & 2.847 & 1.619 & 0.565 & -0.501 & 0.527 \\
Ítem 9 & 1 & 7 & 4.933 & 1.486 & -0.601 & 0.122 & 0.574 \\
Ítem 10 & 1 & 7 & 2.668 & 1.648 & 0.788 & -0.381 & 0.534 \\
Ítem 11 & 1 & 7 & 5.503 & 1.370 & -1.015 & 1.047 & 0.408 \\
Ítem 12 & 1 & 7 & 4.973 & 1.509 & -0.614 & 0.267 & 0.452 \\
Ítem 13 & 1 & 7 & 4.245 & 1.649 & -0.327 & -0.492 & 0.577 \\
Ítem 14 & 1 & 7 & 2.920 & 1.667 & 0.617 & -0.640 & 0.536 \\
\hline
\end{tabular}

Seguidamente, se procedió a valorar la validez de constructo del Cuestionario de Autorregulación del Aprendizaje, para lo cual se realizó un análisis factorial exploratorio con el método de mínimos cuadrados no ponderados robustos y el método de rotación Promím, ya que los valores de asimetría y curtosis fueron bajos. Los resultados arrojaron un valor moderado de adecuación muestral, a través de la prueba Kaiser-Meyer-Olkin (KMO; 0.759) y un resultado significativo en la prueba de esfericidad de Bartlett $\left(\mathrm{x}^{2}=818.84 ; \mathrm{gl}=91 ; \mathrm{p}=0.001\right)$. Del análisis factorial practicado se obtuvieron dos factores que explican el $52.7 \%$ de la varianza total de la prueba. 
En la Tabla 2 se puede apreciar que los ítems que saturan en el primer factor son el $2,4,5,7,8,10,13$ y 14 ; que explican el $32.9 \%$ de la varianza total de la prueba y coinciden con el factor Control. Mientras que los ítems que saturan en el segundo factor son el 1, 3, 5, 6, 9, 11 y 12; que explican el 19.8\% de la varianza total de la prueba y coinciden con el factor Autonomía. En ambos casos los factores resultantes son iguales a los reportados por Matos (2009), pero en diferente orden. Además, dado que el ítem 5 saturaba en los dos factores, se decidió eliminarlo, pues resulta ser ambiguo al no discriminar en entre el primer y el segundo factor. Asimismo, los índices de bondad de ajuste obtenidos en el procesamiento estadístico, satisfacen los criterios establecidos en la mayoría de los casos $\left(\chi^{2}=104.675\right.$; $\mathrm{gl}=64(p<.001) ; \chi^{2} / \mathrm{gl}=1.635 ; \mathrm{CFI}=.911 \mathrm{IC} 95 \%[.846, .962] ; \mathrm{GFI}=.954 \mathrm{IC} 95 \%$ $[.937, .973]$; AGFI $=.934$ IC 95\% [.911, .961]; RMSEA= .102 IC 95\% [.074; .117]).

Tabla 2

Matriz de estructura, confiabilidad y correlaciones interfactoriales

\begin{tabular}{|c|c|c|c|}
\hline \multirow{2}{*}{ Ítems } & \multicolumn{2}{|c|}{ Factor } & \multirow{2}{*}{ Total } \\
\hline & 1 & 2 & \\
\hline Ítem 1 & & 0.472 & -- \\
\hline Ítem 2 & 0.632 & & -- \\
\hline Ítem 3 & & 0.788 & -- \\
\hline Ítem 4 & 0.407 & & -- \\
\hline Ítem 5 & 0.372 & 0.366 & Eliminado \\
\hline Ítem 6 & & 0.616 & -- \\
\hline Ítem 7 & 0.873 & & -- \\
\hline Ítem 8 & 0.648 & & -- \\
\hline Ítem 9 & & 0.697 & -- \\
\hline Ítem 10 & 0.602 & & -- \\
\hline Ítem 11 & & 0.628 & -- \\
\hline Ítem 12 & & 0.606 & -- \\
\hline Ítem 13 & 0.440 & & -- \\
\hline Ítem 14 & 0.686 & & -- \\
\hline$\alpha$ & 0.801 & 0.785 & 0.796 \\
\hline $\mathrm{F} 1$ & 1 & 0.338 & 0.868 \\
\hline F2 & & 1 & 0.681 \\
\hline Total & & & 1 \\
\hline
\end{tabular}

Finalmente, se calcularon las correlaciones interfactoriales y de cada factor con el puntaje total de la prueba, sin considerar el ítem 5. En la Tabla 2 se puede apreciar que el factor Control y el factor Autonomía se correlacionan de manera positiva moderada $(\mathrm{r}=0.338)$, mientras que las correlaciones con el total de la prueba fueron más fuertes, con coeficientes de correlación 0.868 y 0.681 , 
respectivamente. Para el cálculo de la confiabilidad, se trabajó con el método de consistencia interna y la prueba Alfa de Cronbach, hallándose coeficientes superiores a 0.7 para cada uno de los factores y para el total de la prueba (Tabla 2). Por ello se puede decir que las puntuaciones de la prueba son confiables.

\section{DISCUSIÓN}

A pesar de que la autorregulación del aprendizaje es un concepto ampliamente investigado desde diferentes vertientes teóricas en varios países de habla hispana (Rosário, Pereira, Högemann, Nunes, Figueiredo, Núñez, Fuentes y Gaeta, 2014), en el Perú son pocos los estudios sobre dicho constructo. Algunos de ellos han reportado su relación con la autoeficacia académica (Alegre, 2014) o han destacado su papel en el aprendizaje cooperativo a través del trabajo en equipo de estudiantes universitarios (Aliaga, Ponce, Bulnes, Gutiérrez, Frisancho, Pereda, Delgado y Torchiani, 2008); otros estudios se han orientado por la valoración psicométrica del Cuestionario de Autorregulación del Aprendizaje que evalúa dicho constructo (Matos, 2009; Chávez y Merino, 2015).

En Arequipa empero, no hay estudios publicados sobre la autorregulación del aprendizaje, por ello, en la presente investigación, se ha sometido a escrutinio psicométrico el Cuestionario de Autorregulación del Aprendizaje validado por Matos (2009) en estudiantes universitarios limeños. Nuestros resultados indican que la prueba tiene buena homogeneidad pues todos los ítems presentaron correlaciones ítem-test superiores a 0.4 , lo que supone que todos los ítems miden el mismo constructo teórico.

Además, se ha confirmado la estructura bifactorial que ha sido reportada en estudios previos (Matos, 2009; Chávez y Merino, 2015), sin embrago; existen diferencias sutiles que conviene señalar. En primer lugar, mientras Matos (2009) reporta la dimensión de Autonomía como el factor 1 y la dimensión Control como el segundo factor, aquí se tiene que el factor 1 corresponde con la dimensión Control y el factor 2 con la dimensión Autonomía. Asimismo, en el análisis psicométrico de Matos (2009) los ítems 5 y 13 presentan cargas factoriales en ambos factores, pero en nuestro caso, ello solo ocurre con el ítem 5, motivo por el cual, dicho ítem fue eliminado.

En ese sentido, un aspecto que puede ayudarnos a explicar estas diferencias, podría recaer en el método de extracción y de rotación empleados, pues en el presente estudio se ha aplicado el método de mínimos cuadrados no ponderados robustos y el método de rotación Promím, mientras que en el estudio de Matos (2009) se ha aplicado el método de extracción de componentes principales y el método de rotación Varimax. De hecho, podemos decir que, dado que hemos utilizado un método factorial más robusto, nuestros datos ajustan mejor y solo se ha eliminado un ítem. Además, los niveles de confiabilidad que hemos obtenido 
son ligeramente superiores que los de Matos (2009), por tanto, podemos decir que el Cuestionario de Autorregulación del Aprendizaje es válida y confiable para los estudiantes universitarios de psicología de la muestra tomada en la ciudad de Arequipa.

Por otro lado, en el análisis psicométrico de Matos (2009) el factor Autonomía es el que explica la mayor cantidad de varianza total de la prueba, es decir, que sería el factor dominante, mientras que, en nuestro caso, el factor Control es el que explica la mayor cantidad de varianza total, ubicándose como el primer factor. Dado que el factor Control hace referencia a reguladores externos del aprendizaje y que factor Autonomía se refiere a reguladores internos (Williams \& Deci, 1996), podría suponerse que, en la muestra de estudiantes arequipeños, la autorregulación del aprendizaje responde más a factores externos que internos, lo cual es consistente con estudios previos, que ya hemos señalado anteriormente (Arias, 2011, 2014; Arias, Zegarra y Justo, 2014).

Nuestros resultados psicométricos, dada la estructura bifactorial resultante, sugieren que debe promoverse el desarrollo de habilidades metacognitivas, estrategias de aprendizaje y la conducta autorregulada entre los estudiantes universitarios (Montgomery, 2008). Sobre las propiedades psicométricas del Cuestionario de Autorregulación del Aprendizaje, si bien cuenta con validez de constructo y confiabilidad; deben realizarse investigaciones sobre diversas cualidades psicométricas como la invarianza, la validez predictiva, convergente y divergente, en muestras representativas de diferentes niveles de enseñanza. El uso adecuado de este instrumento, podría además, propiciar la investigación educativa y el diseño de programas para desarrollar el aprendizaje autónomo de los estudiantes. Se puede concluir por tanto, que el Cuestionario de Autorregulación del aprendizaje es una prueba válida con una estructura bifactorial e índices adecuados de confiabilidad, sin embargo, estos resultados son solo preliminares, dado el tamaño de la muestra.

\section{Agradecimientos / Acknowledgments:}

Los autores agradecemos a los participantes de este estudio, pues sin su colaboración no hubiera posible su ejecución.

\section{Fuentes de financiamiento / Funding:}

Recursos propios.

\section{Rol de los autores / Authors roles:}

WLAG: realizó el marco teórico, el diseño metodológico y la revisión general.

RR: realizó el análisis estadístico de los datos.

KDCC: realizó la discusión y participó en la recolección de datos. 


\section{Aspectos éticos / Legales ; Ethics / Legals:}

Se ha respetado lo establecido por las normativas éticas que regulan el ejercicio profesional (Código de Ética del Colegio de Psicólogos del Perú). Se procuró la protección de la confidencialidad de la información personal e institucional, asegurando el anonimato de las personas e instituciones involucradas en la muestra.

\section{Conflicto de intereses / Competing interests:}

Los autores manifestamos no haber tenido ningún conflicto de intereses al realizar esta investigación y articulo.

\section{REFERENCIAS}

Alegre, A. A. (2014). Autoeficacia académica, autorregulación del aprendizaje y rendimiento académico en estudiantes universitarios. Propósitos y Representaciones, 2(1), 79-100. doi: 10.20511/pyr2014.v2n1.54

Aliaga, J., Ponce, C., Bulnes, M., Gutiérrez, V., Frisancho, A., Pereda, J., Delgado, E. y Torchiani, R. (2008). Aprendizaje en equipo: evaluación y relación con el aprendizaje autorregulado. Revista Peruana de Psicología, 2(2), 255-268.

Arias, W. L. (2008). Fundamentos del aprendizaje. Arequipa: Vicarte.

Arias, W. L. (2011). Estilos de aprendizaje en estudiantes universitarios y sus particularidades en función de la carrera, el género y el ciclo de estudios. Learning Styles Review, 8, 112-135.

Arias, W. L. (2014). Estilos de aprendizaje e inteligencia en estudiantes universitarios de Arequipa, Perú. Journal of Learning Styles, 7(14), 88-107.

Arias, W. L. y Vilca, J. I. (2007). Estudio neuropsicológico de cinco pacientes con lesiones prefrontales. Revista de Psicología (Universidad Católica de Santa María), 4, 74-86.

Arias, W. L., Zegarra, J., \& Justo, O. (2014). Estilos de aprendizaje y metacognición en estudiantes de psicología de Arequipa. Liberabit, 20(2), 267-279.

Castillo, I., Balaguer, I. y Duda, J. L. (2001). Perspectivas de meta de los adolescentes en el contexto académico. Psicothema, 13(1), 79-86.

Chávez, G. y Merino, C. (2015). Validez estructural de la Escala de Autorregulación del Aprendizaje para estudiantes universitarios. Revista Digital de Investigación en Docencia Universitaria, 9(2), 65-76. doi: 10.19083/ridu.9.453

Contreras, I. y Lozano, A. (2012). Aprendizaje autorregulado como competencia para el aprovechamiento de los estilos de aprendizaje en alumnos de educación superior. Revista de Estilos de Aprendizaje, 10, 114-126.

Covarrubias, M. A., Gómez, J. T. y Estrevel, L. B. (2005). Los estilos de interacción paterna-materna y el desarrollo de la autorregulación afectiva en el niño: Una perspectiva histórico cultural. Revista Cubana de Psicología, 22(1), 62-67. 
Escurra, L. M. (2002). Reflexión y aprendizaje de los estudiantes del quinto de secundaria de Lima. Revista de Investigación en Psicología, 5(2), 55-71.

Escurra, L. M. (2006). Análisis psicométrico del Inventario de Estrategias de Aprendizaje y estudio en estudiantes universitarios de psicología de Lima metropolitana. Perso$n a, 9,127-170$.

Escurra, L. M. (2011). Análisis psicométrico del Cuestionario de Honey y Alonso de Estilos de Aprendizaje (CHAEA) con los modelos de la teoría clásica de los test y de Rasch. Persona, 14, 71-109.

Fernandes, F., Marín, F. J. y Urquijo, S. (2010). Relación entre los constructos autocontrol y autoconcepto en niños y jóvenes. Liberabit, 16(2), 217-226.

Fuente, J. de la, Pichardo, M. C., Justicia, F., \& Berbén, A. (2008). Enfoques de aprendizaje, autorregulación y rendimiento en tres universidades europeas. Psicothema, 20(4), 705-711.

García, J. L., Sánchez, C., Jiménez, M. A. y Gutiérrez, M. (2012). Estilos de aprendizaje y estrategias de aprendizaje: Un estudio en discentes de postgrado. Revista de Estilos de Aprendizaje, 10, 65-78.

Gargurevich, R. (2008). La autorregulación de la emoción y el rendimiento académico en el aula: El rol del docente. Revista Digital de Investigación en Docencia Universitaria, 4(1), 1-13.

Gargurevich, R. y Matos, L. (2010). Propiedades psicométricas del cuestionario de autorregulación emocional adaptado para el Perú. Revista de Psicología (Universidad César Vallejo), 12, 192-215.

Gómez, I. y Luciano, M. C. (1991). Autocontrol en niños: Un estudio experimental sobre dos procedimientos en la adquisición de conductas de espera. Psicothema, 3(1), 25-44.

González, E. (2013). Concepciones implícitas del aprendizaje en estudiantes de psicología de universidades públicas. Revista de Psicología (Universidad César Vallejo), 15(2), 183-193.

IBM. (2011). IBM SPSS Statistics (Version 20) [programa informático en CD-ROM].

Kaplan, R. M., \& Saccuzzo, D. P. (2006). Pruebas psicológicas. Principios, aplicaciones y temas. México: Thomson.

Lamas, H. (2008). Aprendizaje autorregulado, motivación y rendimiento académico. $\mathrm{Li}$ berabit, 14, 15-20.

Lambert, Z. V., Wildt, A. R., \& Durand, R.M. (1991). Approximating confidence intervals for factor loadings. Multivariate Behavioral Research, 26(3), 421-434. doi: 10.1207/ s15327906mbr2603_3

Livia, J. y Ortiz, M. (2014). Construcción de pruebas psicométricas: Aplicaciones a las ciencias sociales y de la salud. Lima: Universidad Nacional Federico Villarreal.

Lorenzo-Seva, U., \& Ferranzo, P. (2017). Factor (Version 10.5.03). Tarragona, España: Universidad Rovira i Virgili. Descargado de: http://psico.fcep.urv.es/utilitats/factor/ 
Magnusson, D. (1969). Teoría de los tests. México: Trillas.

Martínez-Fernández, R. (2007). Concepción de aprendizaje y estrategias metacognitivas en estudiantes universitarios de psicología. Anales de Psicología, 23(1), 7-16.

Matos, L. (2009). Adaptación de dos cuestionarios de motivación: Autorregulación del Aprendizaje y Clima de Aprendizaje. Persona, 12, 167-185.

Merchán, I. M., Bermejo, M. L., \& González, J. D. (2014). Eficacia de un Programa de Educación Emocional en Educación Primaria. Revista de Estudios e Investigación en Psicología y Educación, 1(1), 91-99. doi: 10.17979/reipe.2014.1.1.30

Meza, A. (2013). Estrategias de aprendizaje. Definiciones, clasificaciones e instrumentos de medición. Propósitos y Representaciones, 1(2), 193-213. doi: 10.20511/ pyr2013.v1n 2.48

Montero, I. y León, O. G. (2002). Clasificación y descripción de las metodologías de investigación en Psicología. Revista Internacional de Psicología Clínica y de la Salud, 2(3), 503-208.

Montgomery, W. (2008). Teoría, investigación y aplicaciones clínicas del autocontrol. Revista de Investigación en Psicología, 11(2), 215-225.

Parra, J., Cerda, C., López-Vargas, O., \& Saiz, J. L. (2014). Género, autodirección del aprendizaje y desempeño académico en estudiantes de pedagogía. Educación y Educadores, 17(1), 91-107.

Revel, A. y González, L. (2007). Estrategias de aprendizaje y autorregulación. Revista Latinoamericana de Estudios educativos, 3(2), 87-98.

Rodríguez, M. C. y Vázquez, E. (2013). Fortalecer estilos de aprendizaje para aprender a aprender. Revista de Estilos de Aprendizaje, 11, 19-37.

Rodríguez, W. C. (2013). El lugar de la afectividad en la psicología de Vygotski: Reflexividad histórica y reivindicación. Propósitos y representaciones, 1(2), 105-129. doi: 10.20511/pyr2013.v1n2.35

Rosário, P., Pereira, A., Högemann, J., Nunes, A. R., Figueiredo, M., Núñez, J. C., Fuentes, S. y Gaeta, M. L. (2014). Autorregulación del aprendizaje: Una revisión sistemática en revistas de la base Scielo. Universitas Psychologica, 13(2), 781-797. doi: 10.11144/Javeriana. UPSY13-2.aars

Thornberry, G. (2003). Relación entre motivación de logro y rendimiento académico en alumnos de colegios limeños de diferente gestión. Persona, 6, 197-216

Thornberry, G. (2008). Estrategias metacognitivas, motivación académica y rendimiento académico en alumnos ingresantes a una universidad de Lima metropolitana. Persona, 11, 177-193.

Timmerman, M. E., \& Lorenzo-Seva, U. (2011). Dimensionality Assessment of Ordered Polytomous Items with Parallel Analysis. Psychological Methods, 16, 209-220.

Valle, A., Barca, A., González, R. y Núñez, J. C. (1999). Las estrategias de aprendizaje. Revisión teórica y conceptual. Revista Latinoamericana de Psicología, 31(3), 425-461. 
Valle, A., González, R., Barca, A., \& Núñez, J. C. (2000). Dimensiones cognitivo-motivacionales y aprendizaje autorregulado. Revista de Psicología (Pontifica Universidad Católica del Perú), 14(1), 3-34.

Valle, A., Cabanach, R. G., Rodríguez, S., Núñez, J. C. y González-Pineda, J. A. (2006). Metas académicas, estrategias cognitivas y estrategias de autorregulación del estudio. Psicothema, 18(2), 165-170.

Valle, A., González, R., Núñez, J. C., Suárez, J. M., Piñeiro, I. y Rodríguez, S. (2000). Enfoques de aprendizaje en estudiantes universitarios. Psicothema, 12(3), 368-375.

Vallejos, J., Jaimes, C., Aguilar, E. y Merino, M. (2012). Validez, confiabilidad y baremación del Inventario de Estrategias Metacognitivas en estudiantes universitarios. Revista de Psicología (Universidad César Vallejo), 14(1), 9-20.

Vigotsky, L. S. (1979). Desarrollo de las funciones psicológicas superiores. Barcelona: Editorial Crítica.

Vigotsky, L. S. (1995). Pensamiento y lenguaje. Barcelona: Paidos.

Wertsch, J. V. (1988). Vygotsky y la formación social de la mente. Barcelona: Ediciones Paidós.

Williams, G. C., \& Deci, E. L. (1996). Internalization of biopsychosocial values by medical students: a test of self determination theory. Journal of Personality and Social Psychology, 70(4), 767-779. doi: 10.1037 // 0022-3514.70.4.767 